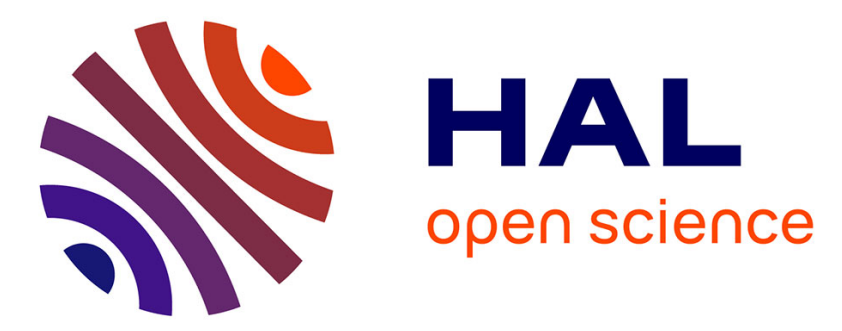

\title{
Changement de phase structural dans RbCdf3 et TlCdf3
}

\author{
M Rousseau, J.Y. Gesland, J. Julliard, J. Nouet, J. Zarembowitch, A.
}

Zarembowitch

\section{To cite this version:}

M Rousseau, J.Y. Gesland, J. Julliard, J. Nouet, J. Zarembowitch, et al.. Changement de phase structural dans RbCdf3 et TlCdf3. Journal de Physique Lettres, 1975, 36 (5), pp.121-124. 10.1051/jphyslet:01975003605012100 . jpa-00231168

\section{HAL Id: jpa-00231168 https://hal.science/jpa-00231168}

Submitted on 1 Jan 1975

HAL is a multi-disciplinary open access archive for the deposit and dissemination of scientific research documents, whether they are published or not. The documents may come from teaching and research institutions in France or abroad, or from public or private research centers.
L'archive ouverte pluridisciplinaire HAL, est destinée au dépôt et à la diffusion de documents scientifiques de niveau recherche, publiés ou non, émanant des établissements d'enseignement et de recherche français ou étrangers, des laboratoires publics ou privés. 


\title{
CHANGEMENT DE PHASE STRUCTURAL DANS $\mathrm{RbCdF}_{3}$ ET TICdF
}

\author{
M. ROUSSEAU, J. Y. GESLAND, J. JULLIARD, J. NOUET \\ Laboratoire de Physique de l'Etat Condensé, Faculté des Sciences, \\ Route de Laval, Le Mans Cedex 72017, France
}

\section{J. ZAREMBOWITCH}

Laboratoire de Chimie de Coordination, E.N.S.C.P. 11, rue Pierre-et-Marie-Curie, 75230 Paris Cedex 05, France

\section{A. ZAREMBOWITCH}

Laboratoire de Recherches Physiques $(*)$, Université Pierre-et-Marie-Curie, 75230 Paris Cedex 05, France

(Reçu le 16 décembre 1974, accepté le 26 février 1975)

\begin{abstract}
Résumé. - Nous avons mis en évidence dans les pérovskites fluorées $\mathrm{RbCdF}_{3}$ et $\mathrm{TlCdF}_{3}$ un changement de phase structural., La symétrie du cristal devient quadratique $(c / a>1)$ pour $T<T_{\mathrm{c}}$ $\left(T_{\mathrm{c}}=124 \mathrm{~K}\right.$ pour $\mathrm{RbCdF}{ }_{3}$ et $T_{\mathrm{c}}=191 \mathrm{~K}$ pour $\left.\mathrm{TlCdF}_{3}\right)$. Les anomalies d'élasticité observées sur $C_{44}$ sont compatibles avec un mécanisme de distorsion par rotations des octaèdres. L'indexation des raies de surstructure du spectre de rayons $X$ ainsi que l'analyse du spectre de diffusion Raman nous permettent de retenir le groupe $\mathrm{D}_{4 \mathrm{~h}}^{18}$ pour la phase basse température. Deux modes de basse fréquence ont dans ces composés un comportement de modes mous.
\end{abstract}

Abstract. - We have shown that a tetragonal distortion with $c / a>1$ occurs at $124 \mathrm{~K}$ in $\mathrm{RbCdF}_{3}$ and at $191 \mathrm{~K}$ in $\mathrm{TlCdF}_{3}$. The anomalous behaviour of the elastic constant $C_{44}$ is consistent with a slight rotation of the $\mathrm{CdF}_{6}$ octahedra. The study of both $\mathrm{X}$-ray superstructure reflexions and Raman scattering spectrum allows us to retain the space group $\mathrm{D}_{4 \mathrm{~h}}^{18}$ for the low temperature phase. Two low frequency modes show the behaviour of soft modes.

1. Introduction. - Les composés $\mathrm{AMF}_{3}$ où $\mathrm{A}$ est un ion monovalent et $\mathrm{M}$ un ion divalent présentent un intérêt tout particulier car ils offrent un choix très varié de matériaux isolants de structure pérovskite dont les propriétés sont étroitement liées aux choix des ions $\mathrm{A}$ et $\mathbf{M}$. De nombreuses études ont été effectuées sur des cristaux de cette série et en particulier sur $\mathrm{KMnF}_{3}[1,2]$ qui présente d'une part, à $184 \mathrm{~K}$, une transition antiferrodistorsive liée à la rotation des octaèdres $M_{6}$ et d'autre part, à $88 \mathrm{~K}$, une transition liée à l'ordre antiferromagnétique. Dans le cadre de nos études sur la dynamique du réseau cristallin des fluopérovskites [3], nous avons cherché à élaborer des composés diamagnétiques présentant des transitions purement antiferrodistorsives.

L'analyse des conditions de stabilité de la structure pérovskite, à partir des constantes de force et des rayons ioniques permet d'envisager a priori l'existence d'un changement de phase dans les fluopérovs-

$\left(^{*}\right)$ Laboratoire associé au C.N.R.S. $n^{0} 71$. kites diamagnétiques qui présentent un faible taux d'encombrement de l'ion $\mathrm{A}^{+}$dans le site délimité par les douze ions fluors voisins. De telles conditions sont vérifiées pour les fluopérovskites du cadmium $\mathrm{RbCdF}_{3}$ et $\mathrm{TlCdF}_{3}$ que nous avons synthétisées sous forme de monocristaux et étudiées par diverses techniques physiques.

2. Elaboration des matériaux. - Les composés $\mathrm{RbCdF}_{3}$ et $\mathrm{TlCdF}_{3}$, à fusion congruente, sont d'abord synthétisés par réaction directe en phase solide à une température de $600^{\circ} \mathrm{C}$ pendant 12 heures après déshydratation sous vide secondaire à $200^{\circ} \mathrm{C}$. Une analyse radiocristallographique permet l'identification du produit obtenu dont la synthèse cristalline est alors effectuée par la méthode BridgmanStockbarger en creuset de platine scellé. La croissance est réalisée par déplacement du creuset à très faible vitesse $(1 \mathrm{~cm} / 24 \mathrm{~h})$ dans un four dont le gradient de température est maintenu constant $(8 \% \mathrm{~cm})$. Les solides obtenus présentent de larges zones monocristallines dans lesquelles il est possible de tailler 
des parallélépipèdes dont les orientations et les dimensions sont adaptées aux mesures physiques envisagées. Ainsi, nous avons pu effectuer les mesures de constantes élastiques et des études de diffusion Raman sur des échantillons monocristallins de dimensions respectives

$$
11 \times 12 \times 14 \mathrm{~mm}^{3} \text { et } 3 \times 5 \times 4 \mathrm{~mm}^{3} \text {. }
$$

3. Analyse radiocristallographique. - L'étude des paramètres cristallins effectuée sur diffractomètre à compteur nous a permis de mettre en évidence un abaissement de symétrie dans $\mathrm{RbCdF}_{3}$ et dans $\mathrm{TlCdF}_{3}$. L'examen des différentes raies du spectre montre l'existence d'une déformation quadratique dans ces deux composés. En effet, les raies hhh ne sont pas affectées alors que les raies hhl sont dédoublées, l'intensité d'une des raies étant double de l'autre. Les raies hkl donnent naissance à 3 raies de même intensité.

Nous avons étudié sur la raie 400 l'évolution du paramètre $c / a$ en fonction de la température. Les positions relatives des deux raies affines permettent de montrer que le rapport $c / a$ est supérieur à l'unité. Le dédoublement est effectif loin de la transition, par contre au voisinage de $T_{\mathrm{c}}$ une décomposition graphique permet de séparer les deux raies et de déduire $c / a$.

Pour déterminer avec précision la température de quadratisation, nous avons suivi l'évolution de la raie 521 dont la largeur à mi-hauteur est très sensible aux variations de $c / a$. Nous avons ainsi obtenu les températures de transition suivantes :

$$
\begin{aligned}
& T_{\mathrm{c}}=124 \pm 1 \mathrm{~K} \text { pour } \mathrm{RbCdF}_{3} \\
& T_{\mathrm{c}}=191 \pm 1 \mathrm{~K} \text { pour } \mathrm{TlCdF}_{3}
\end{aligned}
$$

Le franchissement de la transition par températures décroissantes et par températures croissantes ne s'est pas accompagné d'hystérésis.

Les figures 1 et 2 représentent les variations du paramètre $a$ dans la phase cubique et des paramètres $a$ et $c$ dans la phase quadratique pour $\mathrm{RbCdF}_{3}$ et $\mathrm{TlCdF}_{3}$. Il est à noter que l'amplitude de la distorsion est maximale dans $\mathrm{TlCdF}_{3}$ et atteint $1,3 \%$ à $4,2 \mathrm{~K}$.

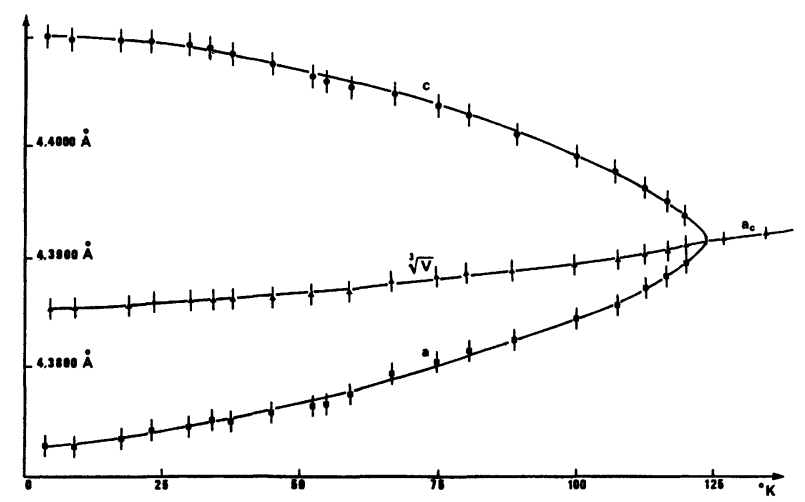

FIG. 1. - Variation, avec la température, des paramètres quadratiques $a$ et $c$, du paramètre moyen quadratique et du paramètre cubique $a_{\mathrm{c}}$ dans $\mathrm{RbCdF}_{3}$.

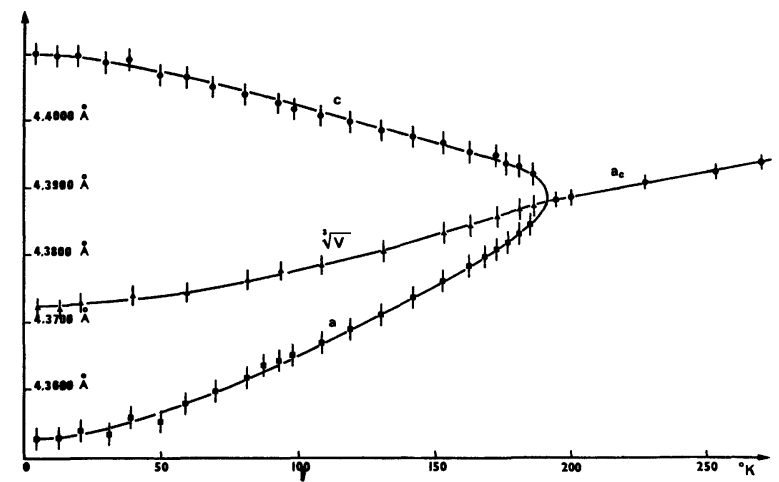

Fig. 2. - Variation, avec la température, des paramètres quadratiques $a$ et $c$, du paramètre moyen quadratique et du paramètre cubique $a_{\mathrm{c}}$ dans $\mathrm{TlCdF}_{3}$.

Nous avons comparé les variations du paramètre moyen quadratique $\sqrt[3]{a^{2} c}$ à celles des paramètres $a$ et $c$. A la transition l'absence de discontinuité de volume indique que le changement de phase est probablement du second ordre.

4. Constantes élastiques, mécanisme de la distorsion. - Nous avons étudié, en fonction de la température, les vitesses des ondes ultrasonores longitudinales et transversales pour les directions $|100|,|110|$ et $|111|$ par une méthode de résonance en ondes entretenues et par une méthode d'impulsions. Les

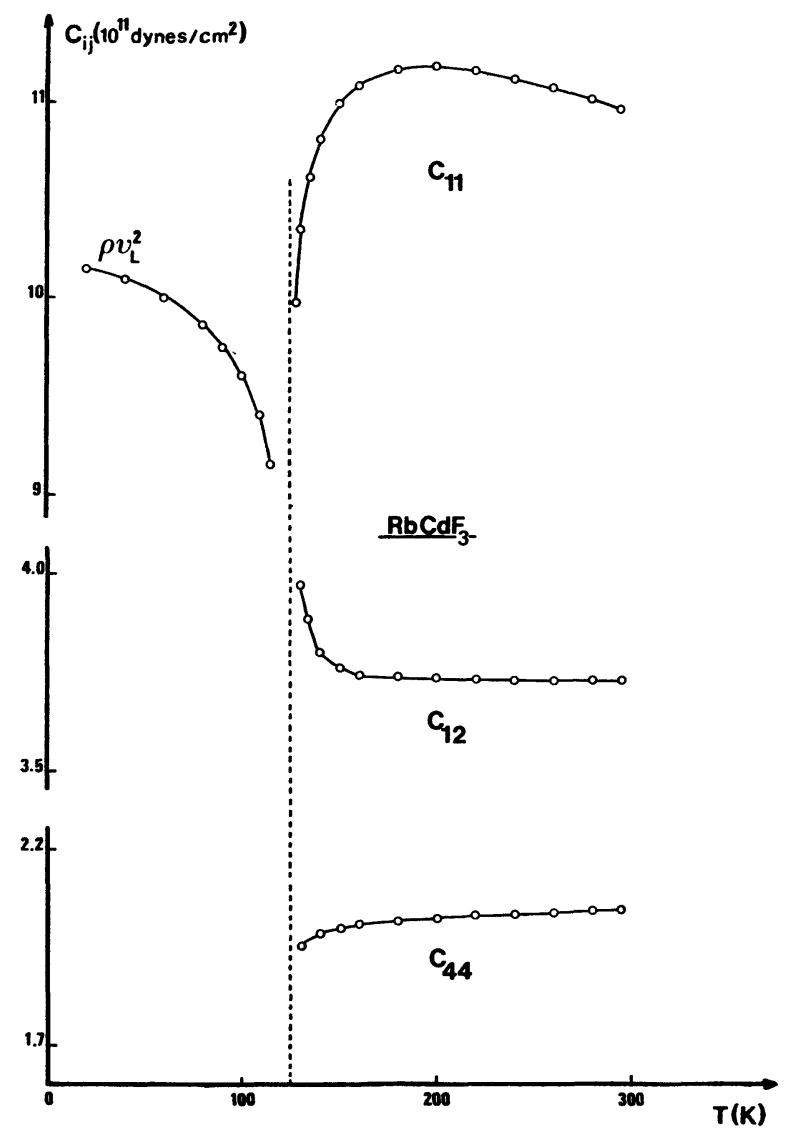

FIG. 3. - Variation des constantes élastiques de $\mathrm{RbCdF}_{3}$ en fonction de la température. 


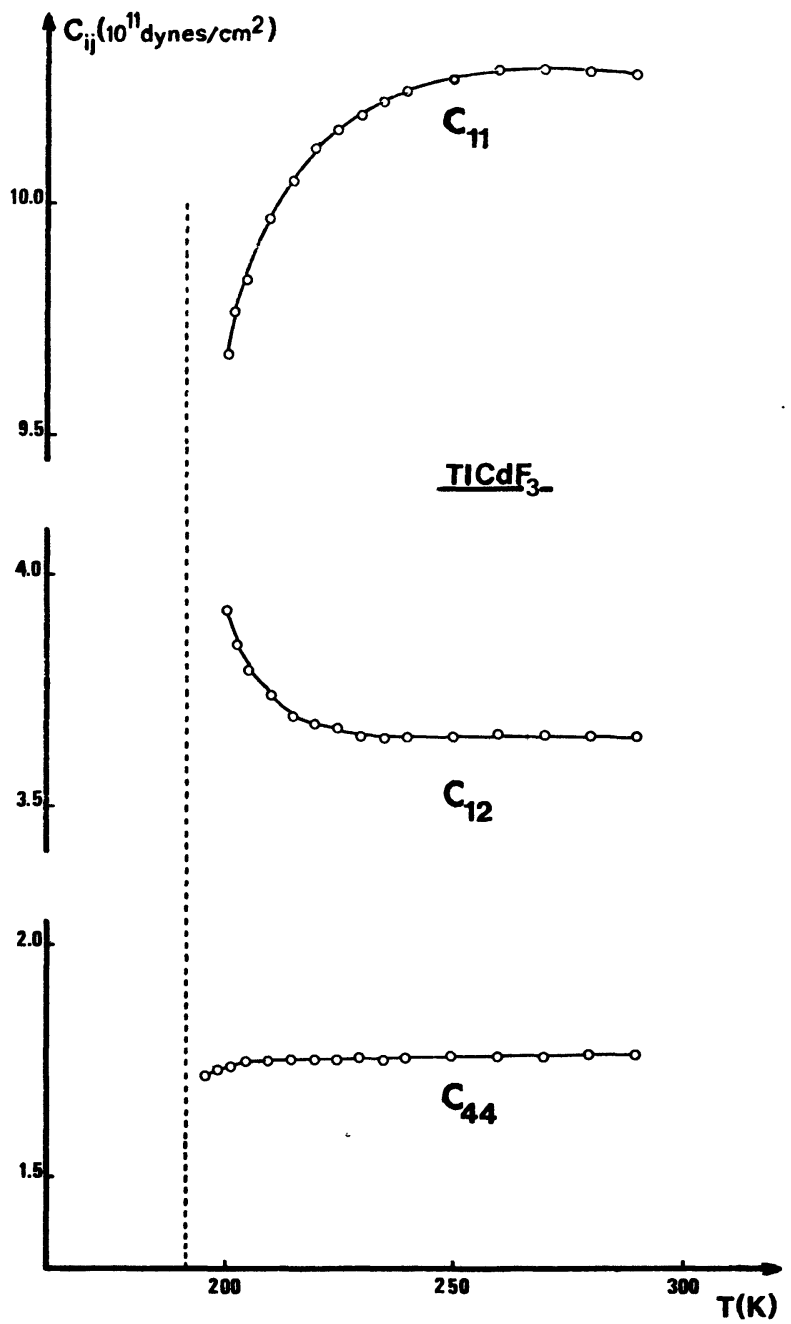

FIG. 4. - Variation des constantes élastiques de $\mathrm{TlCdF}_{3}$ en fonction de la température.

constantes élastiques $C_{11}, C_{12}$ et $C_{44}$ qui s'en déduisent sont représentées sur les figures 3 et 4 , respectivement pour $\mathrm{RbCdF}_{3}$ et $\mathrm{TlCdF}_{3}$.

En utilisant un modèle simplifié [3] dérivé du modèle de Cowley [5] proposé pour $\mathrm{SrTiO}_{3}$, il est possible d'interpréter les variations de $C_{44}$ par un ramollissement de la force de rappel qui s'oppose à la rotation des octaèdres $\mathrm{CdF}_{6}$ [4]. De plus, le faible encombrement stérique des ions $\mathrm{Rb}$ et $\mathrm{Tl}$ ne limite que faiblement ce mouvement de rotation. Nous sommes donc amenés à retenir l'hypothèse d'une distorsion par rotations d'octaèdres.

La phase distordue étant quadratique avec $c / a>1$, dans la classification de Glazer [6] qui envisage toutes les structures distordues dérivées de la structure pérovskite par rotation des octaèdres, seuls les groupes spatiaux $D_{4 h}^{5}$ et $D_{4 h}^{18}$ peuvent être retenus.

5. Discussion. - Dans le but de trancher entre ces deux groupes d'espace, nous avons étudié, sur poudre, les raies de surstructure qui apparaissent dans le spectre de rayons $\mathrm{X}$ pour $T<T_{\mathrm{c}}$. Elles s'indexent toutes sur la base d'une maille quadratique $a \sqrt{2}, a \sqrt{2}, 2 c$ et sont compatibles avec les règles d'extinction du groupe $D_{4 h}^{18}$. Les mêmes conditions expérimentales révèlent un comportement identique pour $\mathrm{KMnF}_{3}$ dont le groupe d'espace $\left(\mathrm{D}_{4 \mathrm{~h}}^{18}\right)$ a déjà été déterminé dans la phase basse température [1].

Par ailleurs, l'étude des spectres de diffusion Raman confirme le choix du groupe d'espace retenu : dans l'hypothèse du groupe $\mathrm{D}_{4 \mathrm{~h}}^{5}$, quatre modes normaux appartenant aux représentations $B_{1 \mathrm{~g}}, \mathrm{~B}_{2 \mathrm{~g}}$, $A_{1 g}, E_{g}$ sont actifs en diffusion alors que sept modes se répartissant en $1 A_{1 g}+3 E_{g}+2 B_{1 g}+1 B_{2 g}$ sont à prévoir pour le groupe d'espace $D_{4 h}^{18}$.

Au dessous de

$$
\begin{aligned}
& T_{\mathrm{c}}=191 \mathrm{~K} \text { pour } \mathrm{TlCdF}_{3} \\
& T_{\mathrm{c}}=124 \mathrm{~K} \text { pour } \mathrm{RbCdF}_{3},
\end{aligned}
$$

on observe en effet l'apparition de 7 raies dont les fréquences à $40 \mathrm{~K}$ figurent dans le tableau I. L'étude de la polarisation de ces raies permet de les attribuer sans ambiguïté aux modes actifs en diffusion dans le groupe $D_{4 h}^{18}$.

TABLEAU I

Fréquences des modes actifs en diffusion Raman dans $\mathrm{RbCdF}_{3}$ et $\mathrm{TlCdF}_{3}$ à $40 \mathrm{~K}$

\begin{tabular}{lrr} 
& $\mathrm{RbCdF}_{3}$ & \multicolumn{1}{c}{$\mathrm{TlCdF}_{3}$} \\
$\mathrm{E}_{\mathrm{g}}$ & - & - \\
$\mathrm{A}_{1 \mathrm{~g}}$ & $27 \mathrm{~cm}^{-1}$ & $16 \mathrm{~cm}^{-1}$ \\
$\mathrm{E}_{\mathrm{g}}$ & $72 \mathrm{~cm}^{-1}$ & $101 \mathrm{~cm}^{-1}$ \\
$\mathrm{~B}_{1 \mathrm{~g}}$ & $78 \mathrm{~cm}^{-1}$ & $46 \mathrm{~cm}^{-1}$ \\
$\mathrm{E}_{\mathrm{g}}$ & $82 \mathrm{~cm}^{-1}$ & $47 \mathrm{~cm}^{-1}$ \\
$\mathrm{~B}_{1 \mathrm{~g}}$ & $189 \mathrm{~cm}^{-1}$ & $139 \mathrm{~cm}^{-1}$ \\
$\mathrm{~B}_{2 \mathrm{~g}}$ & $196 \mathrm{~cm}^{-1}$ & $153 \mathrm{~cm}^{-1}$ \\
\hline
\end{tabular}

Quand la température s'élève de 40 à $124 \mathrm{~K}$, deux modes sont considérablement affectés dans $\mathrm{RbCdF}_{3}$ : l'un des modes $\mathrm{E}_{\mathrm{g}}$ dont on peut suivre la diminution de fréquence de $27 \mathrm{~cm}^{-1}$ à $40 \mathrm{~K}$ jusqu'à $10 \mathrm{~cm}^{-1}$

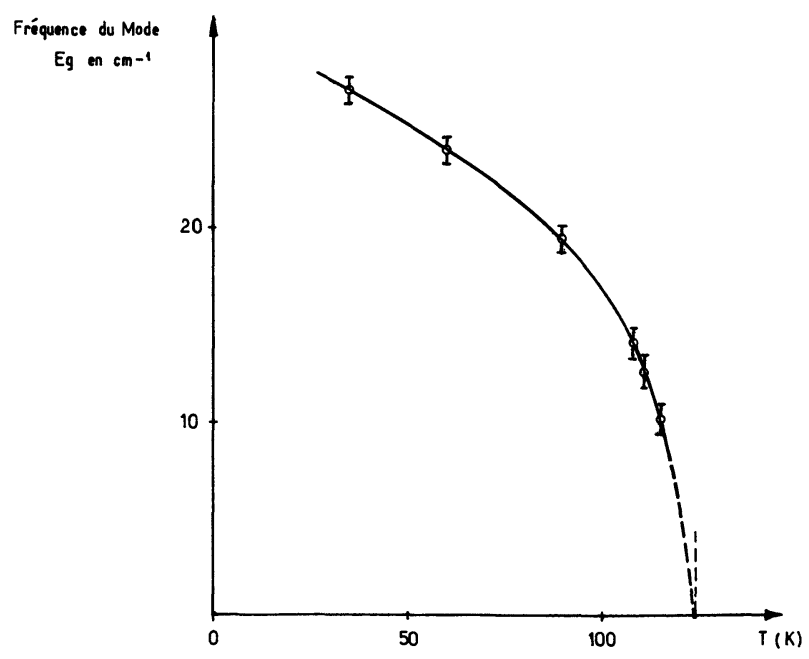

Fig. 5. - Evolution avec la température du mode $\mathrm{E}_{\mathrm{g}}$ de plus basse fréquence dans $\mathrm{RbCdF}_{3}$. 
à $115 \mathrm{~K}$ (Fig. 5) et le mode $\mathrm{A}_{1 \mathrm{~g}}$ qui s'atténue fortement et voit également sa fréquence diminuer. Le spectre du cristal de $\mathrm{TlCdF}_{3}$ présente des évolutions comparables entre 40 et $191 \mathrm{~K}$ pour les raies $\mathrm{E}_{\mathrm{g}}$ et $\mathrm{A}_{1 \mathrm{~g}}$ homologues des précédentes. Cependant la raie $\mathrm{E}_{\mathrm{g}}\left(16 \mathrm{~cm}^{-1}\right.$ à $\left.40 \mathrm{~K}\right)$ dont l'atténuation est considérable ne peut être suivie dans un vaste domaine de température car elle vient élargir la raie de diffusion Rayleigh dont elle ne peut être distinguée [4].
Ces modifications des spectres montrent clairement l'existence de modes mous dus (comme dans $\mathrm{KMnF}_{3}$ [7] et $\mathrm{SrTiO}_{3}$ [8]) à la condensation du mode $\mathrm{R}_{25}$ de la phase cubique.

Remerciements. - Nous remercions Mesdames N. Lenain et D. Simons, Messieurs C. Caray, G. Hamel et G. Niesseron pour leur assistance technique.

\section{Bibliographie}

[1] Minkiewicz, V. J., Fujil, Y. and Yamada, Y., J. Phys. Soc. Japan 28 (1970) 443.

[2] Gesi, K., Axe, J. D., Shirane, G. and Linz, A., Phys. Rev. B 5 (1972) 1933.

[3] Rousseau, M., Nouet, J. and Zarembowitch, A., J. Phys. Chem. Solids 35 (1974) 921.

[4] Rousseau, M., Gesland, J. Y., Julliard, J., Nouet, J., ZaremBOWITCH, A. et J. (à paraître).
[5] Cowley, R. A., Phys. Rev. 134 (1964) 981.

[6] Glazer, A. M., Acta Cryst. B 28 (1972) 3384.

[7] Lockwood, D. J. and Torrie, B. H., J. Phys. C. : S Mid State Phys. 7 (1974) 2729.

[8] Fleury, P. A., Scott, J. F. and Worlock, J. M., Phys. Rev. Lett. 21 (1968) 16. 